\title{
Less is more: «Top 5 list» établie par des étudiants en médecine
}

\author{
Romain Bonnet ${ }^{\mathrm{a}}$, Mathieu Laurencet ${ }^{\mathrm{a}}$, Jean-Michel Gaspoz ${ }^{\mathrm{a}, \mathrm{b}}$, Omar Kherad ${ }^{\mathrm{c}}$

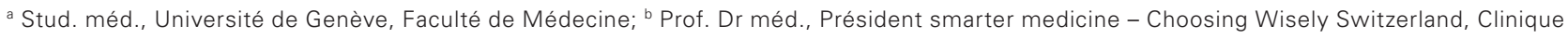 \\ des Grangettes; ' PD Dr méd., MPH, Chef du service de Médecine Interne, Hôpital de La Tour, Genève
}

\section{Introduction}

La campagne "Choosing Wisely», lancée par l’American Board of Internal Medicine, vise à aider les médecins et les patients à mener des discussions sur des tests, des traitements et des procédures qui pourraient s'avérer inutiles et potentiellement néfastes pour le patient [1]. La philosophie de cette campagne repose sur le principe du "less is more» qui est une invitation à reconnaître qu'un excès de traitements peut parfois amener plus de risques que de bénéfices au patient. Ainsi, des "top five lists» d'interventions médicales à faible valeur ajoutée ont vu le jour dans différentes spécialités médicales pour faire face à la hausse des coûts de la santé et encourager les différents partenaires à favoriser une médecine raisonnable. L'objectif initial n'était pas nécessairement de réduire les coûts, mais plutôt de tordre le cou à certaines idées reçues, selon lesquelles davantage de soins apporteraient toujours un bénéfice au patient.

En Suisse, suite à l'élan de la campagne "smarter medicine» lancée par la Société Suisse de Médecine Interne Générale (SSMIG), une nouvelle association de soutien, "smarter medicine - Choosing Wisely Switzerland", a vu le jour en juin 2017 [2]. Cette association est constituée par l'Association suisse des sciences médicales (ASSM) et la SSMIG, mais également par la Fédération suisse des associations professionnelles du domaine de la santé (FSAS) et des associations de défense des intérêts des patients et des consommateurs. Elle devrait donner un nouvel élan à l'initiative. En effet, tout le monde s'accorde à dire que la publication de listes de traitements inutiles ne représente qu'un début. Plusieurs barrières se dressent sur le chemin de l'implémentation de ces mouvements, parmi lesquelles l'illusion thérapeutique des médecins, l'attente des patients et un système fragmenté où le prestataire de soins est payé à l'acte.

Il convient donc de prendre toutes les mesures possibles pour modifier le comportement médical, en intégrant des feedbacks éducatifs, des contrôles par les pairs et la mise en place de stratégies de communication orientées patients pour modifier l'opinion publique.

\section{Formation pré- et postgraduée des médecins}

Parmi toutes ces différentes mesures, la formation préet postgraduée des médecins semble avoir un impact positif sur la diminution d'actes inutiles. Plus cette éducation est précoce, plus l'effet se fait ressentir à long terme. Des études ont en effet démontré que les médecins formés dans des centres soucieux de l'économicité tendent à pratiquer une médecine plus rationnelle après leur internat, ce qui souligne au passage l'importance de la formation dans les centres régionaux et les cabinets médicaux [3, 4].

De par leur position unique dans les unités de soins, les étudiants en médecine représentent un précieux baromètre critique de nos pratiques médicales. Ils ont également une formidable capacité à créer une dynamique sur des causes d'importance commune. Ainsi, les étudiants en médecine canadiens ont été les premiers à publier leur propre liste d'interventions d'actes jugés inutiles pour encourager les médecins formateurs à réfléchir à deux fois avant de prescrire un acte potentiellement inutile [5]. Plus récemment en Suisse, à la demande de médecins impliqués dans le mouvement, les étudiants en médecine de l'Université de Genève ont mis en place un groupe de travail (Smarter Medical Students Geneva), chargé d'élaborer une liste regroupant les interventions de médecine hospitalière à remettre en question de leur point de vue.

\section{Processus de sélection}

Après l'évaluation minutieuse des listes établies par le mouvement "Choosing Wisely», le groupe de travail a sélectionné les 15 recommandations qu'ils estimaient les plus pertinentes en fonction de leur propre expérience clinique. Les étudiants en médecine à l'Université de Genève ont par la suite été priés, au cours de 
Tableau 1

1. Ne pas prescrire d'antibiotique en cas d'infection virale des voies aériennes supérieures (sinusite, pharyngite, bronchite)

2. Eviter l'utilisation de fluoroquinolone comme traitement de première ligne dans les infections urinaires non compliquées chez la femme

3. Ne pas laisser les personnes âgées alitées pendant leur séjour à I'hôpital

4. Ne pas utiliser d'opiö̈de en première ligne comme traitement d'une douleur chronique non cancéreuse

5. Ne pas priver un patient d'un traitement de pénicilline en raison d'une notion d'allergie, sans effectuer un bilan allergologique au préalable

l'été 2017, d'évaluer la liste et de classer les items sur la base de la pertinence clinique en Suisse en intégrant les critères suivants: fréquence de l'intervention, preuves scientifiques, évaluation du rapport bénéfice/risque pour le patient et possibilité d'influencer la pratique médicale. La plupart des participants $(\mathrm{n}=86)$ à l'enquête étaient des stagiaires de $5^{\mathrm{e}}$ et $6^{\mathrm{e}}$ année avec au moins six mois d'exposition clinique. A l'issue de ce classement, une liste des 5 recommandations ayant obtenu les meilleurs scores a pu être établie et publiée [6].

\section{Conclusion}

Cet élégant exercice a permis de révéler que les étudiants en médecine n'étaient en réalité pas très familiers avec le mouvement "Choosing Wisely». Cette jeune génération s'est toutefois montrée très sensible à cette approche axée sur la qualité et sur le concept du «less is more», qui représente non seulement un défi intellectuel, mais aussi une véritable opportunité de promouvoir la médecine durable. Lors des études de médecine, les étudiants sont souvent encouragés à démontrer leur curiosité scientifique et leurs connaissances médicales. Cet environnement universitaire peut amener à une surutilisation des actes médicaux lors de prises en charge. Les campagnes "Choosing Wisely" cherchent à modifier ces comportements en rappelant aux étudiants l'importance d'analyser les actes médicaux sous l'angle de leur risque potentiel.

A cet égard, des "less is more cursus» vont voir le jour ces prochaines années, tant dans les facultés de médecine que lors des congrès internationaux. Cela permettra d'assurer une exploitation rationnelle, économique et éthique des ressources médicales tout en respectant parfaitement le principe du «less is more» où la réduction des coûts n'est pas prioritaire, mais peut devenir un effet collatéral positif quand elle converge avec l'intérêt du patient.

\section{Références}

1 http://www.choosingwisely.org

2 http://www.smartermedicine.ch

3 Stammen LA, Stalmeijer RE, Paternotte E, et al. Training physicians to provide high-value, cost-conscious care: A systematic review. JAMA. 2015;314:2384-400.

4 Chen C, Petterson S, Phillips R, Bazemore A, Mullan F. Spending patterns in region of residency training and subsequent expenditures for care provided by practicing physicians for Medicare beneficiaries. JAMA. 2014;312:2385-93.

5 https://choisiravecsoin.org/campaign/espoirs

6 Bonnet R, Laurencet M, Gaspoz JM, Kherad O. Creating a list of low-value health care interventions according to medical students perspective. Eur J Intern Med. 2018 Jun;52:e41-2. 